\title{
Abdominal Aortic Aneurysm Repair by Principle of Exclusion and Aortic Bypass: Single Centre Experience
}

\author{
Sandeep $M^{*}$ and Ramakrishna $P$
}

Department of Vascular Surgery, Nizam's Institute of Medical Sciences, Punjagutta, Hyderabad, India

\begin{abstract}
Purpose: Non-respective treatment of the abdominal aortic aneurysm by proximal and distal ligation of the aneurysm sac (exclusion) combined with aortic bypass known as Debakey Procedure has been previously reported. A 2 year experience with 10 patients undergoing this procedure was reviewed.

Methods: From 2010 to 2012, 10 patients of abdominal aortic aneurysm underwent repair with the retroperitoneal exclusion technique. Preoperative morbidity and mortality, blood loss and transfusion requirements, naturally of the excluded aneurysm sac were all assessed.

Results: The operative mortality rate for patients undergoing exclusion and bypass was $10 \%$ (one out of 10 patients). The incidence of nonfatal preoperative complications was $5 \%$. Blood loss requiring transfusion in the preoperative period measured $600 \mathrm{~mL}$ to $900 \mathrm{~mL}$ on an average. On follow-up, only one out of 10 patients was found to have patent aneurysm sacs as detected by duplex examination which required surgical intervention. No cases of graft infection or aortoenteric fistula have been noted.

Conclusion: Retroperitoneal exclusion and bypass is a viable alternative to traditional open endoaneurysmorraphy in surgery for abdominal aortic aneurysm. Most excluded aneurysm sacs have thrombosis without any long- or short-term complications; however, in a small number of patients delayed rupture of patent aneurysm occurs, thus emphasizing the need for diligent follow-up and appropriate intervention.
\end{abstract}

Keywords: Abdominal aortic aneurysm; Principle of exclusion; Endoaneurysmorraphy

\section{Introduction}

Conventional treatment of thoracic abdominal aortic aneurysms (TAAAs) consists of graft replacement with reattachment of the main aortic branches. Over the past 20 years a multimodal approach has gradually evolved to reduce the trauma of surgery by maximizing organ protection, allowing experienced surgical centres to have better outcomes.

Historically, the techniques to treat patients with aneurysms of the aorta or peripheral arteries included ligation proximal to the aneurysm, excision and over-sewing of the neck of the aneurysm, placement of wire into the sac to effect thrombosis, aneurysm wrapping with an external support, and endoaneurysmorraphy. Approximately 40 years ago, the modern era of thoracic aortic surgery was heralded by Lam and Aram, who used an aortic homograft to replace the descending thoracic aorta for aneurismal disease [1]. In 1953, Debakey and Cooley reported the first successful replacement of a descending thoracic aortic aneurysm using a synthetic vascular graft [2] and the surgical approach to thoracoabdominal aortic aneurysms has been carefully evaluated by Crawford et al. [3-7].

However, mortality and morbidity associated to TAAA open repair remain significant. Hybrid repair, consisting of open aortic disbranching and revascularization followed by endovascular exclusion of the aneurysm, may extend the indications of TAAA repair to highrisk patients that cannot benefit from surgery; however results are still under evaluation.

\section{Materials and Methods}

In between 2010 to 2012, 10 out of 25 patients of thoracic Abdominal Aortic aneurysm underwent repair with the retroperitoneal exclusion technique. Preoperative morbidity and mortality, blood loss and transfusion requirements, natural history of the excluded aneurysm sac were all assessed in those patients.

The disease characteristics of those patients were grouped as A. Juxta renal aneurysm with solitary functioning left kidney B. Juxta renal aneurysm with functioning left kidney, SMA. IMA occlusion C: Juxta renal AAA with occluded celiac, SMA.

The patients were evaluated with Spiral CT angiogram suggestive of Juxta renal aortic aneurysm and supra celiac extension (Figures 1 and 2). Under general anaesthesia, the patient was placed in right lateral position with appropriate precautions for pressure areas and monitoring of the vital parameters. The midline abdominal incision is extended to the lateral chest wall along the 6 the intercostals space. The diaphragm was incised all along the costal attachment. The descending aorta was isolated carefully taking care of the intercostals vessels. Retro peritoneal mobilisation of left renal and the iliac vessels was carried out preparing the bed for the distal anastomosis. Proximal side clamp was placed to the descending thoracic aorta and end to side graft aorta anastomosis was carried out. The two limbs of the Dacron graft were anastomosed to the bilateral common iliac arteries in end to side

*Corresponding author: Sandeep M, Assistant Professor, Department of Vascular Surgery, Nizam's Institute of Medical Sciences, Punjagutta, Hyderabad, India, Tel: +91 9948769761; E-mail: sanusandeep@gmail.com

Received January 17, 2016; Accepted February 04, 2016; Published February 12,2016

Citation: Sandeep M, Ramakrishna P (2016) Abdominal Aortic Aneurysm Repair by Principle of Exclusion and Aortic Bypass: Single Centre Experience. J Vasc Med Surg 4: 253. doi:10.4172/2329-6925.1000253

Copyright: (c) 2016 Sandeep M, et al. This is an open-access article distributed under the terms of the Creative Commons Attribution License, which permits unrestricted use, distribution, and reproduction in any medium, provided the original author and source are credited. 
Citation: Sandeep M, Ramakrishna P (2016) Abdominal Aortic Aneurysm Repair by Principle of Exclusion and Aortic Bypass: Single Centre Experience. J Vasc Med Surg 4: 253. doi:10.4172/2329-6925.1000253

Page 2 of 3

Pre operative angiogram

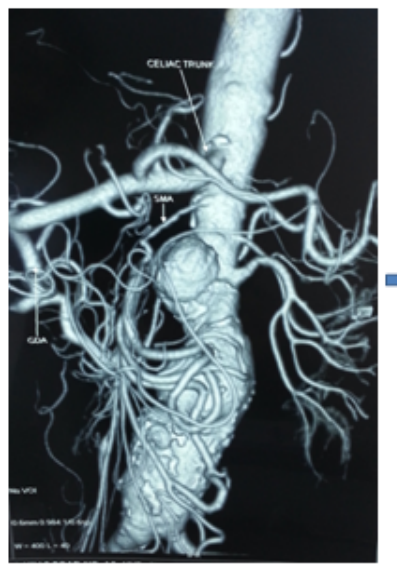

\section{Post operative angiogram}

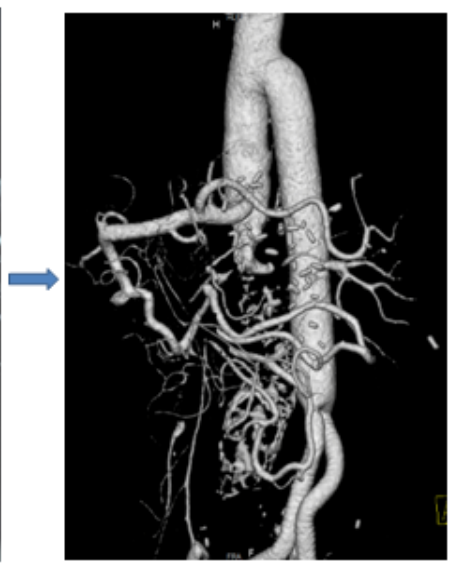

Figure 1: Spiral CT angiogram.
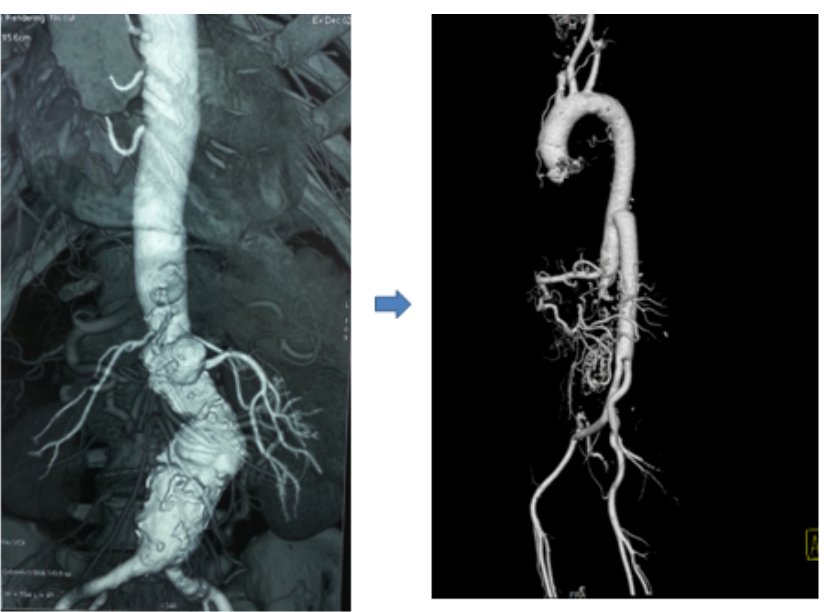

Figure 2: Juxta renal aortic aneurysm and supra celiac extension.

fashion. The circulation was established in torso above and torso below without encountering organ ischemia.

The patients having renal arteries arising from the aneurysm need radionuclide Venogram to appropriately plan for revascularization of individual renal arteries. In addition, mesenteric circulation was also checked by selective mesenteric angiogram before reimplantation. Similar to the disbranching procedure, selective visceral perfusion of renal or mesenteric arteries were then carried out within the warm ischemic time (Figures 3 and 4). The patients were discharged around $10^{\text {th }}$ day post op with uneventful recovery without neuro vascular deficit (Figure 5).

\section{Results}

The patients selected for the exclusion and aortic bypass were between 35 to 55 years of age, in this part of the world, World health organization (WHO) reports that the average longevity of life is around 65 years. Considering Per capita income, life expectancy, risk of rupture of aneurysms, surgery still fares cast effective management for the patients of thoraco- abdominal aneurysms. The symptomatic patients usually present with pain in the back and epigastric region with fullness of the abdomen. The average aneurysm size ranged between $6.7 \mathrm{~cm}$ to $11.8 \mathrm{cms}$ in transverse diameter. 4 out of 10 patients were diabetic and 6 were found to be hypertensive. The patients selected were having near normal renal function tests. 7 patients had "ever smoked" found to have mild to moderate chronic obstructive lung disease.

The average stay in the hospital ranged between 7-12 days for all the patients none of the patients had post operative renal dysfunction requiring dialysis support, however, transient oliguria with altered Kidney function was seen in almost all cases for 1-2 days, which recover spontaneously with adequate hydration and electrolyte correction. Chest tube drain is placed in all cases with thoraco abdominal incision, which usually settles down in 5-7 days. We have not encountered any case of Post operative paraplegia, bowel necrosis, limb ischemia, peri operative MI, sepsis, serial USG follow up is being done to monitor the aneurysm rupture, In 9 out of 10 cases, the aneurysm has shrunk in size with intra mural thrombosis. In one out of 10 cases, the patient had

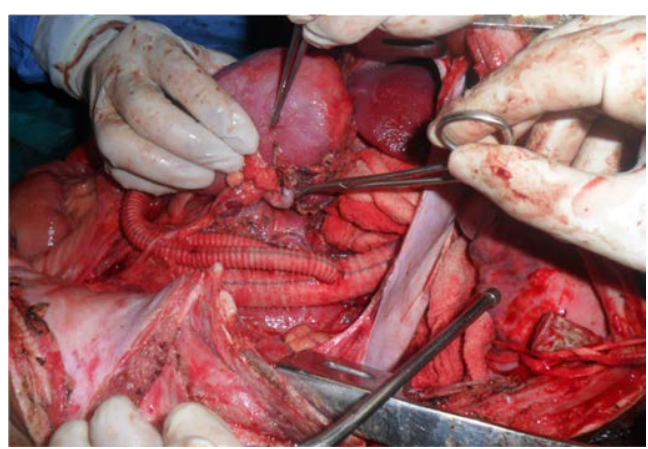

Figure 3: Disbranching procedure, selective visceral perfusion of renal or mesenteric arteries.

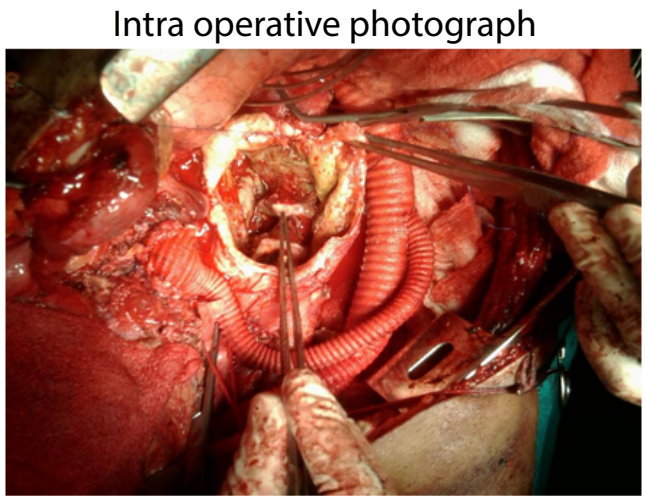

Figure 4: Intra-operative photograph.

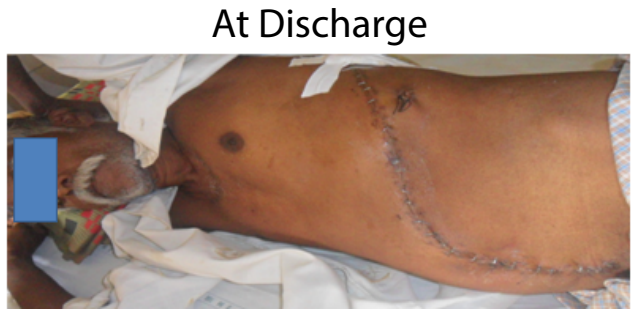

Figure 5: The patients were discharged without neuro vascular deficit. 
persistent pain abdomen that required exploration of the aneurysm. We lost a single case on account of free rupture of aneurysm into the peritoneal cavity.

\section{Discussion}

One of the first successful repairs of a thoracoabdominal aortic aneurysm (TAAA) in the United States was reported in 1955 by Etheredge [8]. Utilizing a 5-mm aortic shunt, an in situ aortic homograft repair of an extent IV aneurysm was performed via a thoracoabdominal incision, this included anastomoses of the celiac and superior mesenteric arteries. That same year, Charles Rob, an English surgeon, also reported on his experience of 33 abdominal aortic aneurysms, six of which required lower thoracic aortic clamping approached via a thoracoabdominal incision. Rob's report predated Etheredge's manuscript, and he is credited with the first description of a TAAA repair [9]. Cooley and DeBakey's initial report of a descending thoracic aortic aneurysm (likely an extent I) approached via a thoracoabdominal incision in 1953 predated Etheredge's manuscript as well [2], but this repair did not involve direct manipulation of the abdominal visceral arteries and thus Etheredge and Robb remain the first surgeons to describe such a technique. Shortly thereafter, Debakey reported on four cases similar to Etheredge's patient utilizing aortic homograft as the conduit, concluding that aortic shunting was imperative to success [10]. His subsequent report in 1965 included 42 patients in whom knitted Dacron grafts were utilized as the initial shunt and then converted to the formal conduit by stepwise side branch anastomoses of the visceral arteries [11]. Most of these initial reports involved aneurysmectomy which prolonged operative times and led to significant morbidity, and Crawford is credited with pioneering the technique of an intra-aortic anastomosis after longitudinal division of the sac. In addition, he also described the technique of a single pedicled visceral segment anastomosis including the celiac, superior mesenteric and right renal arteries, followed by a left renal anastomosis with sequential reperfusion after the completion of each [3]. His results were superb with only one death in 23 consecutive cases, a mortality rate that is rarely achieved in modern practice. Along with the utilization of cardiopulmonary bypass, hypothermic circulatory arrest, and cerebrospinal fluid drainage, Crawford's approach most resembles contemporary techniques performed at major centres today.

The alternate method of management of thoraco abdominal aortic aneurysm includes division of the aorta, with suture closure of the distal aorta and outflow vessels (exclusion of the aneurysm), and end-to-end proximal to distal bypass. There exists a concern that the excluded blood filled aneurysm may not thromboses or may be the source of late sepsis. During an 8-year period, 280 abdominal aortic aneurysms (urgent and elective) were treated by exclusion of the abdominal aortic aneurysm sac and bypass via the poster lateral retroperitoneal approach. Mean age was 70 years (range, 44 to 88), with 217 men and 63 women. Preoperative CT scanning and aortography were performed to assess arterial anatomy. Seventy tube grafts and 260 bifurcation grafts were used. Thirty-day mortality rate was $4 \%$. Estimated blood loss was $731 \pm 52 \mathrm{~mL}$; mean transfusion requirements were $456 \pm 82 \mathrm{~mL}$. The minor complication rate was $6 \%$, and it is of great interest that there were no cases of ischemic colitis requiring colectomy. Aneurysm sacs thrombosis except in two anti coagulated patients who required further treatment. No late infections occurred. Five-year bypass patency rate was $98 \%$ [12].

\section{Conclusion}

The advantages of the procedure applying the principles of exclusion of the aneurysm sac with aortic bypass include a) Less blood loss b) Lesser incidence of organ ischemia c) No need for cardio pulmonary bypass d) Safer dissection, however, extensive retroperitoneal dissection, morbidity of thoracotomy, and post op pain are the short term pitfalls of the procedure, The advantages of the procedure in terms of mortality benefit and safety clearly outweigh the disadvantages. The result obtained in our institution is encouraging enough to conduct the procedure in a large scale to match the international numbers. The procedure is cost effective compared to the endovascular exclusion techniques.

\section{Ethical Approval}

Being a retrospective study with recommended procedure adapted for the management of the patients, Ethical committee approval is not obtained. This article does not contain any experimental studies with human participants or animals performed by any of the authors.

\section{References}

1. Lam CR, Aram HH (1951) Resection of the descending thoracic aorta for aneurysm; a report of the use of a homograft in a case and an experimental study. Ann Surg 134: 743-752.

2. DeBakey ME, Cooley DA (1953) Successful resection of aneurysm of thoracic aorta and replacement by graft. J Am Med Assoc 152: 673-676.

3. Crawford ES (1974) Thoraco-abdominal and abdominal aortic aneurysms involving renal, superior mesenteric, celiac arteries. Ann Surg 179: 763-772.

4. Crawford ES, Snyder DM, Cho GC, Roehm JO Jr (1978) Progress in treatment of thoracoabdominal and abdominal aortic aneurysms involving celiac, superior mesenteric, and renal arteries. Ann Surg 188: 404-422.

5. Crawford ES, Crawford JL, Safi HJ, Coselli JS, Hess KR, et al. (1986) Thoracoabdominal aortic aneurysms: preoperative and Intraoperative factors determining immediate and long-term results of operations in 605 patients. $J$ Vasc Surg 3: 389-404.

6. Crawford ES, Coselli JS, Svensson LG, Safi HJ, Hess KR (1990) Diffuse aneurysmal disease (chronic aortic dissection, Marfan, and mega aorta syndromes) and multiple aneurysm. Treatment by subtotal and total aortic replacement emphasizing the elephant trunk operation. Ann Surg 211: 521537.

7. Dake MD, Miller DC, Semba CP, Mitchell RS, Walker PJ, et al. (1994) Transluminal placement of endovascular stent-grafts for the treatment of descending thoracic aortic aneurysms. N Engl J Med 331: 1729-1734.

8. Etheredge SN, Yee J, Smith JV, Schonberger S, Goldman MJ (1955) Successful resection of a large aneurysm of the upper abdominal aorta and replacement with homograft. Surgery 38: 1071-1081.

9. Rob C (1955) The surgery of the abdominal aorta and its major branches. Ann R Coll Surg Engl 17: 307-318.

10. DeBakey ME, Creech O Jr, Morris GC Jr (1956) Aneurysm of thoracoabdominal aorta involving the celiac, superior mesenteric, and renal arteries; report of four cases treated by resection and homograft replacement. Ann Surg 144: 549 573

11. DeBakey ME, Crawford ES, Garrett HE, Beall AC Jr, Howell JF (1965) Surgical considerations in the treatment of aneurysms of the thoraco-abdominal aorta. Ann Surg 162: 650-662.

12. Shah DM, Chang BB, Paty PS, Kaufman JL, Koslow AR, et al. (1991) Treatment of abdominal aortic aneurysm by exclusion and bypass: an analysis of outcome. J Vasc Surg 13: 15-20. 